\title{
Neutron Small-Angle Scattering from Palladium Alloys*
}

(Extended Abstract only) By B. H. VerBeEk, Kamerlingh Onnes Laboratory, Leiden, The Netherlands, C. VAN DiJK, Netherlands Energy Research Foundation ECN, Petten, The Netherlands, B. D. RaINFord, Blackett Laboratory, Imperial College, London SW7 2BZ, England and A. P. Murani, Institut Laue-Langevin, Grenoble, France

(Received 3 November 1977; accepted 15 February 1978)

The dependence of the neutron small-angle scattering intensities on an applied magnetic field and on the temperature has been investigated for a number of dilute PdFe and PdMn alloys. Critical magnetic scattering was observed at small $q$ values over a wide temperature range. Applying a magnetic field of $4 \mathrm{kOe}$ was usually sufficient to suppress the critical scattering completely, so that from the anisotropy of the residual magnetic scattering proper values for the giant moments could be deduced.

Ferromagnetism in dilute alloys of $\mathrm{Pd}$ with $\mathrm{Co}, \mathrm{Fe}$, and $\mathrm{Mn}$ has long been the subject of many investigations (Nieuwenhuys, 1975). The large observed magnetic moment per impurity atom has made these alloys classical examples of 'giant-moment' systems, and the mechanism of giantmoment formation is a problem of theoretical interest. The localized magnetic impurity interacts with the nearby $\mathrm{Pd}$ $3 d$ electrons, inducing a magnetic polarization cloud around the impurity moment, which may extend to several ångstroms. The experimental evidence for this spatial extension in $\mathbf{P d F e}$ and $\mathbf{P d C o}$ in the ferromagnetic phase was provided more than 10 years ago by Low \& Holden (1966) who employed diffuse neutron scattering using the difference counting technique. Here a magnetic field applied parallel to the scattering vector can be used to separate the magnetic contribution from the total scattering intensity.

Similar neutron studies were performed on PdMn alloys $(c \leq 0.5$ at. $\%$ ) (de Pater, van Dijk \& Nieuwenhuys, 1975) which were investigated in the paramagnetic phase. The results of these measurements showed an enhanced forward scattering which made an extrapolation for $q \rightarrow 0$ impossible,

* This work is part of the research programme of the Foundation FOM supported by ZWO and TNO.

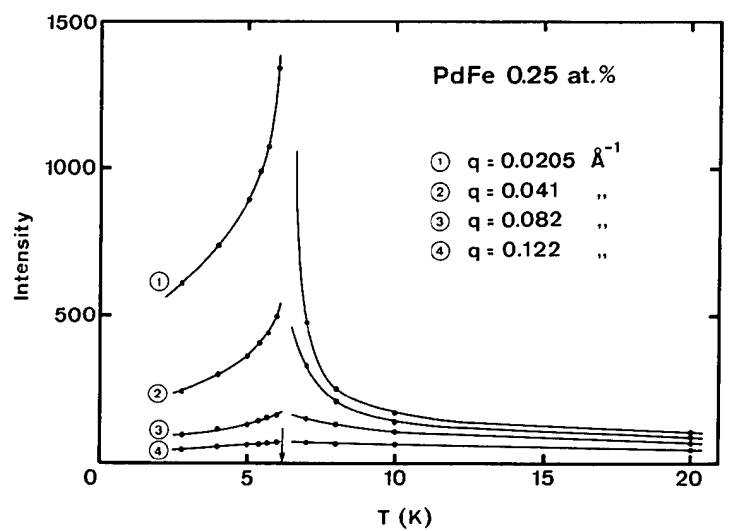

Fig. 1. Neutron scattering intensive vs temperature for PdFe 0.25 at. $\%$ for various $q$ values. corresponding to the giant moment value of $7.5 \mu_{B}$. In order to investigate this small- $q$ behaviour further, extensive measurements were performed at the small-angle scattering spectrometer D11A of the Institute Laue--Langevin in Grenoble.

Experimental data were obtained for PdFe $(0 \cdot 25,0.5,1$ and 2 at. \%) and PdMn $(0 \cdot 25,0.5$ and $2 \cdot 1$ at. $\%)$ over a wide temperature interval $(2.8<T<100 \mathrm{~K})$ and with applied magnetic fields ranging from 0 to $4 \mathrm{k} 0 \mathrm{e}$.

The magnetic scattering of PdFe 0.25 at. $\%$ and PdMn 2.1 at. $\%$ as function of temperature (Figs. 1 and 2) demonstrates clearly that in these random dilute alloys pronounced critical scattering occurs at a well defined temperature. A careful investigation of the critical behaviour in PdMn has been performed by Murani (1977).

The wide temperature range in which the critical contribution is observed can be explained qualitatively by inspection of the $q$-dependent susceptibility as given in a mean-field approximation

$$
\gamma(q)=\frac{\not c}{r_{1}^{2}\left(q^{2}+\kappa_{1}^{2}\right)},
$$

where for $T>T_{c} r_{1}^{2}=\frac{1}{6} J^{(2)} / J(0)=r_{n}^{2} / 6$ for nearest neighbours and $\kappa_{1}^{2}=\left(T-T_{c}\right) r_{1}^{-2} / T_{c}, \psi_{c}$ is the Curie susceptibility, and $\kappa_{1}$ is the inverse correlation length. For a dilute system one can use for $r_{n}$ the mean distance between the impurity atoms, which is relatively large, so that the inverse correlation length remains relatively small over a wide temperature range.

From these results the observed enhancement of the forward scattering in diffuse scattering experiments (de Pater, van Dijk \& Nieuwenhuys, 1975; Verbeek, Amundsen \& van Dijk, 1977) may be very well explained as originating from critical contributions at small $q$ values.

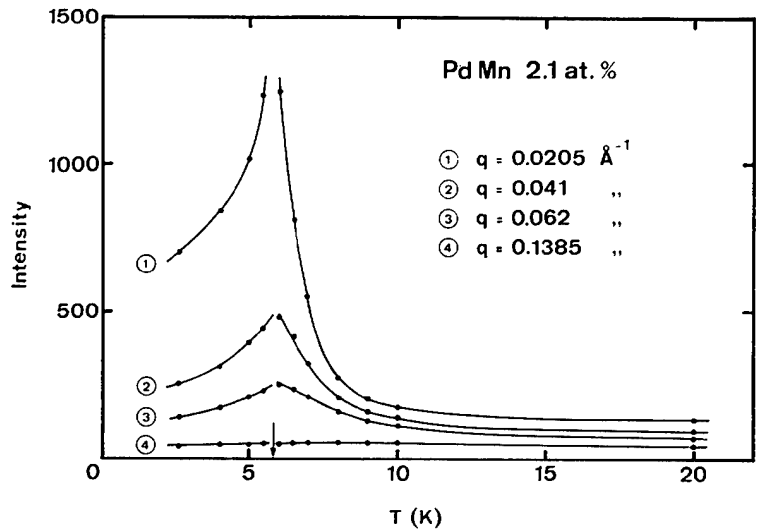

Fig. 2. Neutron scattering intensity is temperature for PdMn $2 \cdot 1$ at. $\%$ for various $q$ values. 
The magnetic-field dependence of the critical scattering of PdMn 2.1 at. $\%$ is shown in Fig. 3. A magnetic field isotropically reduces the critical scattering and with a field of $3.6 \mathrm{k0e}$ the scattering can almost completely be suppressed.

The anisotropy of the magnetic small-angle scattering in a

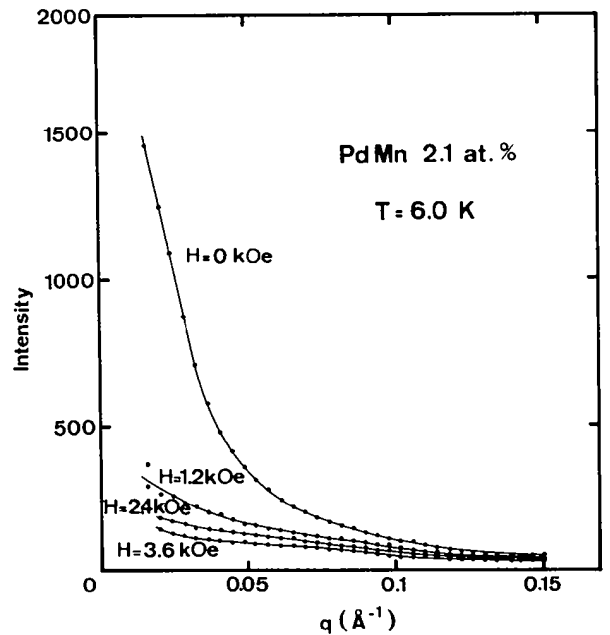

Fig. 3. Magnetic-field dependence on the small-angle scattering in PdMn 2.1 at. $\%$ in the critical temperature region. magnetic field of $4 \mathrm{kOe}$ was measured for a number of PdFe alloys at $3 \cdot 2 \mathrm{~K}$. The magnetic field suppresses the critical scattering, so the observed anisotropy is solely due to the diffuse scattering by the magnetic giant moment. From the analysis of the anisotropic data magnetic cross sections were obtained which are less than the extrapolated values deduced by Low \& Holden (1966), suggesting that critical scattering may have also played a role in their experiments. The corresponding magnetic moment per impurity is about $8 \mu_{B}$ which is smaller than the high-field magnetization value of $10-11 \mu_{B}$ for $\mathrm{Fe}$. The question now arises whether these values can be compared in view of the peculiar low-field magnetization data of these alloys in the ferromagnetic phase (Nieuwenhuys, 1975, p. 542). Magnetization measurements are planned on the same samples and under identical conditions as the neutron experiments were performed. A full account of the investigation will be given in the near future in Journal of Physics F.

\section{References}

Low, G. G. \& Holden, T. M. (1966). Proc. Phys. Soc. London, 89, 119-127.

Murani, A. P. (1977). Unpublished work.

Nieuwenhuys, G. J. (1975). Ad'. Phys. 24, 515-591.

Pater, C. J. DE, VAN DiJk, C. \& NieuwenhuYs, G. J. (1975). J. Phy's. F, 5, L 58-L 63.

VerbeeK, B. H., AMUNDSEN, T. \& VAN DiJK, C. (1977). Physica, 86-88B, 482-484.

\title{
J. Appl. Cryst. (1978). 11, 638-641 \\ Magnetic Small-Angle Scattering from Molten Elements Iron, Cobalt, and Nickel
}

By M. WEBER, W. KNOLL and S. STEEB, Max-Planck-Institut für Metallforschung, Institut für Werkstoffwissenschaften, Seestrasse 92, D-7000 Stuttgart 1, Federal Republic of Germany

(Received 3 January 1978; accepted 17 April 1978)

\begin{abstract}
Scattering of neutrons $(\lambda=0.7 \AA)$ from molten iron was carried out at $1600^{\circ} \mathrm{C}\left(T_{\text {melt }}=1536^{\circ} \mathrm{C} ; T_{\text {Curie }}=770^{\circ} \mathrm{C}\right)$ within the region of $q=0 \cdot 2-2 \cdot 0 \AA^{-1}$. A pronounced small-angle scattering effect was observed for $q \leq 1 \cdot 2 \AA^{-1}$, which is explained by magnetic scattering from spin fluctuations. A correlation length $\xi=2 \cdot 7 \AA$ was obtained. The plot of $\log \xi$ versus $\log \left[\left(T-T_{\text {Curie }}\right) / T_{\text {Curie }}\right]$ together with the $\zeta$ values obtained from solid iron shows that all measured $\xi$ values can be described by the law $\xi=2 \cdot 15\left[\left(T-T_{\text {Curie }}\right) / T_{\text {Curie }}\right]^{-0.5}$. Molten cobalt at $1550^{\circ} \mathrm{C}\left(T_{\text {mclt }}=1493^{\circ} \mathrm{C} ; T_{\text {Curic }}=1127^{\circ} \mathrm{C}\right)$ yields a correlation length of $6.3 \AA$. Finally the results from a corresponding experiment on molten nickel at $1500^{\circ} \mathrm{C}\left(T_{\text {melt }}=\right.$ $1454^{\circ} \mathrm{C} ; T_{\text {Curie }}=358^{\circ} \mathrm{C}$ ) are presented. However, the observed scattering effect is very small compared with that from the two other elements.
\end{abstract}

Until now neutron scattering experiments from pure molten transition metals have been performed in the region of momentum transfer $q \geq 1 \AA^{-1}$ (see for example, Waseda \& Suzuki, 1970), which is too large to reveal any significant features concerning small-angle scattering. During the present work experiments were performed down to $q=0.2 \AA^{-1}$ on molten iron, cobalt, and nickel. The experiments were done on the D4 two-axis diffractometer at the Grenoble high-flux reactor with neutrons of wavelength $\lambda=0.7 \AA$ (Institut Laue-Langevin, 1975). Although not specially designed for neutron-scattering studies at small momentum transfer, this instrument provides reliable data down to $q_{\min } \simeq 0 \cdot 2 \AA^{-1}$. This minimum momentum transfer is sufficient for the study of short-range fluctuations, as was shown by investigations on concentration fluctuations in binary molten alloys (Wignall \& Egelstaff, 1967; Zaiss, Steeb \& Bauer, 1976).

The iron samples (purity 99.95\%) were sealed in tubes made of polycrystalline $\mathrm{Al}_{2} \mathrm{O}_{3}$ (inner diameter $0.7 \mathrm{~cm}$, wall thickness $0.05 \mathrm{~cm}$ ), whereas for cobalt and nickel (LABgrade materials from Merck) sapphire tubes $(0.7 \times 0.1 \mathrm{~cm})$ were used.

The measured intensity was corrected for scattering and absorption from the container and the heater as well as for self-absorption, and for multiple and incoherent scattering contributions. A vanadium standard was used to obtain absolute differential scattering cross sections. Fig. 1 shows the result of neutron scattering experiments from two samples 